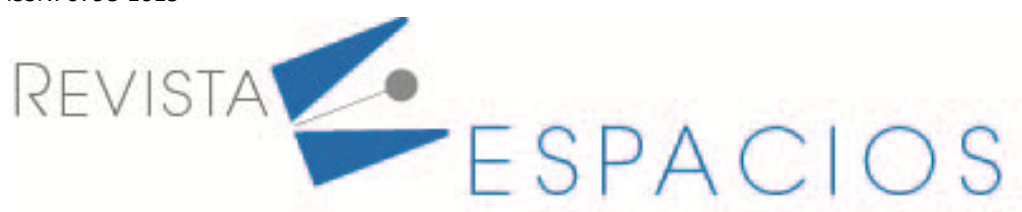

\title{
Sistema de costos basado en actividades - el caso del Hotel Panorama del municipio de Sincelejo, Colombia
}

\section{Cost system based on activities in Hotel Panorama Municipality of Sincelejo Colombia}

\author{
ARROYO, Freddy. ${ }^{1}$ \\ SANTAMARÍA, Álvaro E. ${ }^{2}$ \\ MENDOZA, Arminda. ${ }^{3}$
}

\begin{abstract}
Resumen
El objetivo consistió en proponer un diseño del método de costeo ABC para el Hotel Panorama de Sincelejo, Colombia. La investigación fue no experimental transeccional descriptiva. El método de costeo $A B C$ implementado para el análisis, permitió calcular eficientemente las tarifas de servicios, inventarios, mejorar el control, reducir los costos y, reorientar los presupuestos en períodos más adecuados de tiempo; en síntesis, el metodo $A B C$ en comparación con el método de costeo tradicional, permitió maximiar las utilidades del período analizado.

Palabras clave: método de costeo $A B C$, reducción de costos, centros de costos productivos, centro de costos de apoyo
\end{abstract}

\begin{abstract}
The objective was to propose a design of the $A B C$ costing method for the Hotel Panorama in the city of Sincelejo Colombia. Methodologically, the research was descriptive transectional non-experimental. The $A B C$ costing method made it possible to efficiently calculate service and inventory rates, improve control, reduce unnecessary costs, reorient budgets in more suitable periods, in contrast to the traditional costing method; In short, it allowed maximizing the hotel's profits.

key words: $A B C$ cost method, cost reduction, production cost centers, support cost center
\end{abstract}

\section{Introducción}

La contabilidad de costos dentro de una empresa u organización constituye uno de los aspectos más importantes, ya que se convierten en un instrumento de ayuda para la gerencia, puesto que permite registrar, planear y controlar todas las actividades que se realizan dentro de las mismas. Dicho de otra manera, la contabilidad de costos es un sistema de información que se encarga de la acumulación y del análisis de la información relevante para uso interno de los gerentes en la planeación, control y evaluación de una determinada empresa (Polimeni et al., 1997).

Por otro lado, es importante clarificar que toda la información que se recolecta a través de la contabilidad de costos le sirve a la organización o empresa para tomar futuras decisiones. En este sentido, la contabilidad se

\footnotetext{
${ }^{1}$ Docente. Facultad de Ciencias Económicas y Administrativas. Universidad de Sucre. Email: freddyarroyoa@hotmail.com

2 Profesor Asociado. Facultad de Ciencias Económicas y Administrativas. Universidad de Sucre. Email: alvaro.santamaria@unisucre.edu.co

${ }^{3}$ Docente. Facultad de Ciencias Económicas y Administrativas. Universidad de Sucre. Email: armymendoza_1203@hotmail.com
} 
convierte en una herramienta de apoyo y ayuda, puesto que le permite a la empresa determinar y saber cuánto le cuesta producir o vender un producto, u ofrecer un servicio que esté dentro de la misma, a partir de las actividades que se realizan en la organización.

Sin embargo, para que las empresas no terminen en quiebra y alcancen sus objetivos, es imprescindible que opten por un modelo y método de costeo que implique un esquema de cálculo a aplicar para la determinación de un costo, lo cual permite identificar los gastos concernientes a las diferentes producciones y servicios que ofrece una empresa.

Existen varios métodos de costeo, pero en los últimos años se ha tenido en cuenta un nuevo método para la definición de los costos de los productos y servicios mejor conocidos como ABC; el mismo, se fundamenta en el análisis de uso de recursos y en la organización de los responsables partiendo de las diferentes actividades y sus relaciones. Con base en ésto, el presente trabajo investigativo tiene como objetivo el diseño de un sistema de costos con enfoque en el método $A B C$.

Desde hace varios años el sector empresarial se ha vuelto más competitivo; por ello se hace necesario mejorar los análisis de costos. Los mercados determinan los precios y la rentabilidad se subordina a la eficacia con que se manejen estos costos; de aquí subyace la importancia de éstos, en la ventaja competitiva que pueden llegar a constituir siempre y cuando se tengan bajo control. Para ello, es necesario comprender el nivel de profundidad en relación al proceso de producir o comercializar un bien o servicio, puesto que estos a la final son los objetos de costo.

La hotelería es reconocida como una de las principales actividades generadora de importantes incrementos en los indicadores económicos, debido a que muchas personas constantemente se trasladan de un lugar a otro, ya sea por cuestión de trabajo o turismo; y en el transcurso de estos viajes uno de los principales beneficiarios son los hoteles. Por tal razón, a lo largo y ancho del departamento de Sucre, existen varias empresas que ofrecen servicios de alojamiento, restaurante, gimnasio, lavandería, parqueadero entre otros. Este es el caso del Hotel Panorama, el cual es el centro de estudio de este proyecto investigativo.

El hotel Panorama, se encuentra ubicado en el departamento de Sucre, en el municipio de Sincelejo. Es una organización que ofrece un servicio hotelero, que busca satisfacer las necesidades con calidez y confort, con excelente infraestructura y tecnología. Con relación a los costos relacionados con los servicios que ofrece el hotel, a través de un estudio minucioso se constató que éste, actualmente opera con un método de costeo tradicional, el cual no le permite calcular adecuadamente las tarifas o costos que se ofrecen el hotel. Por otro lado, los gastos fijos son considerados gastos del período y no están siendo absorbidos como objetos de costos, lo cual ocasiona que el sistema de costeo no sea preciso y ponga en riesgo la veracidad de la información.

A partir de lo anterior, se propone el diseño del método de costeo ABC; el cual desde la presunción inicial y conocimmiento de los investigadores, constituye una posible solución al problema encontrado; el cual es considerado un métodos innovador en la gestión empresarial. Teniendo en cuenta lo expresado anteriormente, se plantea la siguiente interrogante: ¿De qué manera el diseño de un sistema de costo desde el método $A B C$ permite calcular mejor las tarifas de los diferentes servicios del Hotel Panorama ubicado en la Ciudad de Sincelejo, Sucre? Con atención al interrogante planteado se considera lo siguiente:

Esta propuesta investigativa presenta un impacto a nivel social, económico y teórico. Social porque se muestra un método de costeo innovador ( $A B C$ ) dinamizador del sector hotelero, dejando a un lado modelos tradicionales. Este método distribuye los costos sobre bases técnicas y contables más razonables, en relación al sistema de costeo tradicional, sobre todo en lo concerniente a la determinación de los costos indirectos que han sido y siguen siendo el aspecto más crítico en la imputación al detalle de los costos (Cruzado, 2017).

Desde el punto de vista económico se considera que otras empresas podrán optar por éste modelo en la contabilidad de sus costos con la seguridad de que mejorarán así sus ingresos al sopesar con seguridad la relación entre gastos y servicios que cada empresa ofrece. Por último, es importante aclarar que ésta propuesta se 
fundamenta en diferentes conceptos, teorías y experiencias favorables por las que han pasado algunas empresas; razón por la cual, se considera su pertinencia. Con relación al estudio de caso seleccionado se considera que el método de conteo $A B C$ generará un impacto favorable, permitiendo al Hotel tomar las medidas pertinentes, si por ejemplo ante un rango de precios determinado, le conviene o no seguir compi dentro del mercado o ajustarse de forma estratégica en atención a su presupuesto.

\subsection{Contetualización teórica}

Actualmente, en el contexto de apertura de la economía, la tecología, y las políticas tributarias, entre otros aspectos, existen factores que demandan de las organizaciones en general y especialmente a los gerentes adoptar nuevas estrategias y herramientas de gestión y control de costos que permitan conocer cuánto les cuesta producir su bienes o servicios, base para fijar sus precios de venta (Garrido et al., 2018).

En ese sentido, el costeo basado en actividades puede definirse como un sistema integral, que reconoce como generador de costos a las actividades que lleva a cabo la empresa, y, por lo tanto, utiliza dichas actividades como base para la asignación de los costos a los distintos productos y/o servicios. Su propósito es proporcionar a los gerentes una herramienta para aumentar la rentabilidad al proveer información basada en hechos, con la cual, se mejoran las 10 decisiones estratégicas, operacionales y de precios, que en forma conjunta determinaran el resultado financiero de una empresa (Laredo et al., 2009).

En este sentido, la contabilidad de costos como herramienta de gestión y control juega un papel fundamental, al realizar una correcta valuación de inventarios $y$, en consecuencia, poder elaborar estados financieros con información precisa para la toma de decisiones. Además de promover procesos que conduzcan al establecimiento de ventajas competitivas sostenibles (Lawrence \& Chad, 2012).

El método de costos $A B C$, como mecanismo para facilitar la alineación con la estrategia oranizacional, la optimización de procesos y la generación de valor, enrruta a las organizaciones a disminuir las tensiones competitivas y a responder a los requerimentos de los clientes, para obtener servicios de mejor calidad y oportunidad; esto está demandado de la alta gerencia implementar soluciones que agregen valor a los servicios para hacerlos más competitivas (Ramírez, 2007).

La principal característica del $A B C$ consiste en centrarse en las actividades que realiza la empresa, en una visión horizontal y siguiendo el flujo de los procesos. Así, el poder descomponer los costos de los productos en las actividades que realiza la empresa, permite analizar en forma más realista y profunda, las posibilidades de reducción de costos. Se pasa de un análisis a nivel muy agregado de gastos-productos a un análisis más detallado y que busca el origen de los costos (Laredo et al., 2009).

Según Paredes (2017) "el sistema de costos por actividades brinda a la empresa conocimiento y manejo de la información, eliminando de su proceso actividades repetitivas o que no generan el valor agregado que busca la empresa para su crecimiento y desarrollo" (p.66).

Los métodos tradicionales utilizados anteriormente, están cediendo el paso a nuevos sistema de costos como el (Sistema de Costos Basado en Actividades) ABC, los cuales parecen estar adaptados en respuesta a las actuales exigencias de mercados los hipercompetitivos, y el cumplimiento de estandares internacionales, evitando producir las distorciones de precios que los sistemas anteriores producían. Es menester entonces, articular el proceso de planeación estratégica y la implementación del sistema de costos $A B C$, como un aspecto clave del mejoramiento interno de los procesos de gestión administrativa, y de presupuestación enfocada por actividades (García \& Serrano, 2006). 


\section{Metodología}

Teniendo en cuenta los objetivos propuestos, este trabajo se enmarca como una investigación no experimental transeccional descriptiva. La investigación descriptiva busca especificar propiedades, características y rasgos importantes de cualquier fenómeno que se analice. En relación a la investigación no experimental las actividades ya han ocurrido y no pueden ser cambiadas por el curso de la investigación, pero si pueden mejorar al aplicar las conclusiones y recomendaciones que se derivan del estudio (Hernández et al., -2016).

Los diseños transeccionales, recolectan datos en un solo momento, en un tiempo único. Su propósito es describir variables, y analizar su incidencia e interrelación en un momento dado, es como tomar una fotografía de algo que sucede (Hernández et al., 2016).

\subsection{Propuesta de diseño aplicando el sistema de costeo $A B C$}

El Hotel Panorama está ubicado en Sincelejo (Sucre). Cuando se revisa lo relativo a su identidad corporativa, el hotel se platea como una organización dedicada a ofrecer el mejor servicio hotelero en Sincelejo, cuyo principal objetivo es satisfacer las necesidades de calidez y confort, mediante un equipo humano competente, infraestructura y tecnología adecuada, para generar un crecimiento organizacional que asegure un mejoramiento continuo.

Al referirse a sus principios y valores la gerencia del hotel afirma que las personas que laboran en sus instalaciones se caracterizan por su amabilidad, alegría, innovación, trabajo en equipo de forma rigurosa y transparente, con un alto sentido de servicio, con la busqueda incesante de la satisfacción de las expectativas de su clientela. Para ello definen varios principios como calidez, confort, innovación, cumplimiento, honestidad y amabilidad.

La calidez para hotel panorama es la carta más importante de presentación con el cliente, se brinda un servicio con gentileza y cordialidad en cada momento de interacción. De esa forma se construyen, puentes de confianza y calidez entre la organización y los clientes.

El confort en el hotel, es concebido como el mantenimiento constante de un enfoque de bienestar para el cliente y para la organización, en ese sentido se ofrecen instalaciones dotadas de los elementos necesarios para generar comodidad, en un ambiente de tranquilidad, agradable para los huéspedes.

En cuanto a la innovación, el hotel busca activamente las ocasiones para mejorar los servicios, afinando detalles en soluciones de problemas y promoviendo mejoras continuas. Aprovecha las oportunidades para actualizar constantemente sus conocimientos, para innovar las instalaciones y los servicios ofrecidos.

El Cumplimiento se trabaja con responsabilidad en pro del cumplimiento de los compromisos delarados a los clientes, satisfaciendo sus necesidades con prontitud, generando confianza y fidelización.

La honestidad es representativa en el hotel, garanza confianza, seguridad e integridad en las relaciones interpersonales. Ofrece conductas de ética, discerniendo claro entre lo que es correcto y lo que es erróneo.

La amabilidad es la primera impresión que se brinda a los clientes, quienes son atendidos con alegría y fervor, poniendo el corazón constantemente a los servicios.

El hotel Panorama ubicado en la ciudad de Sincelejo ofrece las siguientes instalaciones y servicios: restaurante, lavandería, telefonía. Entre sus instalaciones se encuentran: Salón de eventos, alojamiento (habitaciones), distribuidas de la siguiente manera, cuarenta (40) habitaciones sencillas, treinta y dos matrimoniales (32) y cuatro (4) habitaciones triples. 
En la segunda fase se asignan los costos a las actividades pertenecientes a los diferentes centros; de esta forma las actividades se convierten en el núcleo del modelo.

Figura 3

Fase 2 Asigación de Costos

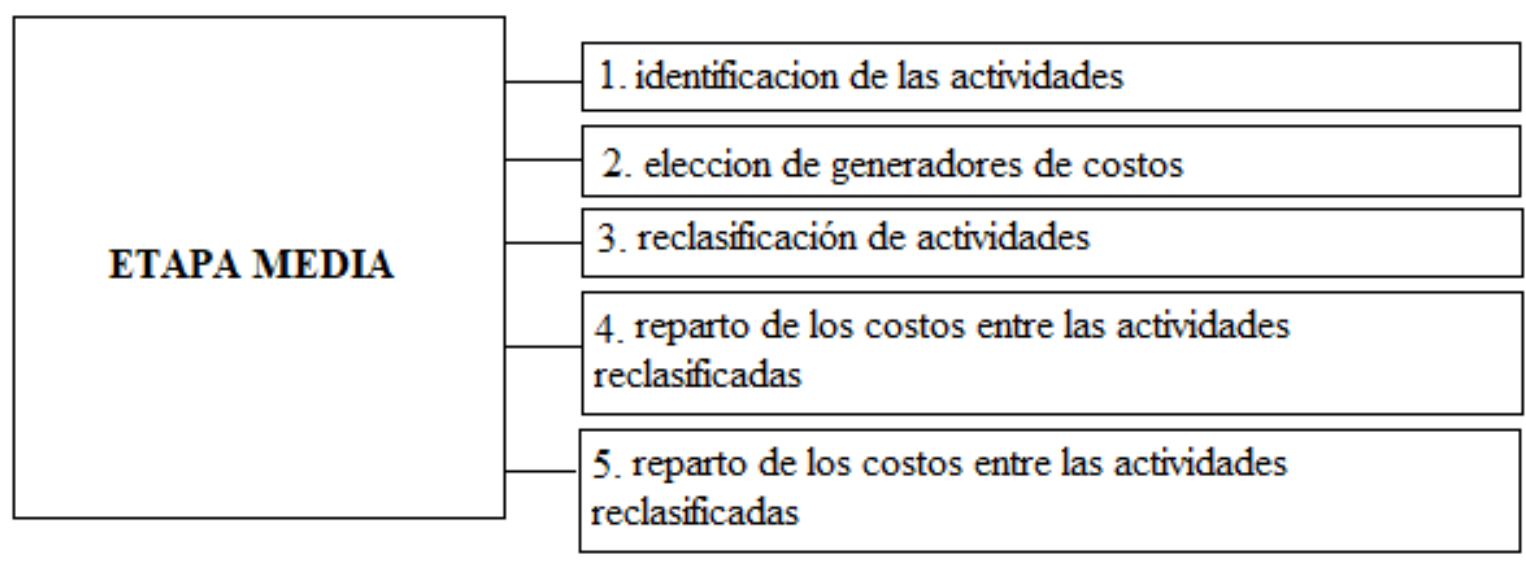

Elaboración propia

Mediante esta etapa se identifican cada una de las actividades que se realizan. El proceso de identificación de las actividades que tienen lugar en los distintos centros constituye una de las etapas más delicadas e importantes. Para ello, uno de los procedimientos que se utilizan es el cuestionario o entrevista entre personas integradas en los centros.

Figura 4

Centros de costos indirecos Identificcaión de actividades realizadas

\begin{tabular}{|c|c|}
\hline Centros de Costos Indirectos & Actividades Realizadas \\
\hline Lavandería Exterior & $\begin{array}{l}\text { Recibir lencería. } \\
\text { Controlar Lencería. } \\
\text { Confeccionar bultos. } \\
\text { Trasladar bultos hacia el camión. } \\
\text { Trasladar bultos desde el camión. } \\
\text { Entregar Lencería. } \\
\text { Lavar Exterior. }\end{array}$ \\
\hline Servicios de Seguridad & Proteger \\
\hline Servicios Técnicos & $\begin{array}{l}\text { Reparar } \\
\text { Dar mantenimiento } \\
\text { Supervisar }\end{array}$ \\
\hline Almacén Servicios Técnicos & $\begin{array}{l}\text { Emitir órdenes de Compras } \\
\text { Transportar } \\
\text { Recepcionar Productos } \\
\text { Despachar Productos }\end{array}$ \\
\hline Limpieza Áreas Públicas & Limpiar \\
\hline Cocina: comedor obrero & $\begin{array}{l}\text { Traer Comestibles } \\
\text { Preparar y cocinar } \\
\text { Servir } \\
\text { Fregar } \\
\text { Limpiar }\end{array}$ \\
\hline
\end{tabular}

Fuete: Elaboración propia 
En esta etapa supone un momento crucial en estos procesos de asignación. En efecto dentro de cada actividad se deberá elegir aquel portador o inductor de costos, que mejor respete la relación causa - efecto entre: Consumo de Recursos - Actividad de Servicios Básicos.

Figura 5

Centros de costos- Inductores de actividades realizadas

\begin{tabular}{|c|c|c|}
\hline Servicios generales & Actividades realizadas & $\begin{array}{c}\text { Generadores de } \\
\text { costos }\end{array}$ \\
\hline Lavandería Exterior & $\begin{array}{l}\text { Recibir lencería. } \\
\text { Controlar Lencería. } \\
\text { Confeccionar bultos. } \\
\text { Trasladar bultos hacia el camión. } \\
\text { Trasladar bultos desde el camión. } \\
\text { Entregar Lencería. } \\
\text { Lavar Exterior. }\end{array}$ & $\begin{array}{l}\text { \# de piezas } \\
\text { \# de bultos } \\
\text { \# de bultos } \\
\text { \# de bultos } \\
\text { \# de bultos } \\
\text { \# de piezas } \\
\text { \# de bultos }\end{array}$ \\
\hline Seguridad & Proteger & \# de postas \\
\hline Servicios Técnicos & $\begin{array}{l}\text { Reparar } \\
\text { Dar mantenimiento }\end{array}$ & $\begin{array}{l}\text { \# de órdenes de } \\
\text { Trabajo } \\
\text { \# de órdenes de } \\
\text { Trabajo }\end{array}$ \\
\hline $\begin{array}{c}\text { Insumos y Servicios } \\
\text { Técnicos }\end{array}$ & $\begin{array}{l}\text { Emitir órdenes de Compras } \\
\text { Transportar } \\
\text { Recepcionar Productos } \\
\text { Despachar Productos }\end{array}$ & $\begin{array}{l}\text { \# de solicitud } \\
\text { \# de transp. } \\
\text { \# de Recepciones } \\
\text { \# de Vales de } \\
\text { Salidas }\end{array}$ \\
\hline Limpieza Áreas Públicas & Limpiar & $M^{2}$ \\
\hline Cocina: comedor obrero & $\begin{array}{l}\text { Traer Comestibles } \\
\text { Preparar y cocinar } \\
\text { Servir } \\
\text { Fregar } \\
\text { Limpiar }\end{array}$ & $\begin{array}{l}\text { \# de comensales } \\
\text { \# de comensales } \\
\text { \# de comensales } \\
\text { \# de comensales } \\
\text { \# de comensales }\end{array}$ \\
\hline
\end{tabular}

Fuete: Elaboración propia

En esta tercera etapa se pretende agrupar las actividades. En efecto se ha visto que dentro de los distintos centros pueden existir idénticas o similares actividades, en este sentido son actividades comunes. Ahora se trata de agregar esas actividades para simplificar los procesos de asignación, con independencia de su localización concreta en un determinado centro. A tal fin, se agrupan las actividades de similares características, formándose así los costos totales de la agrupación de la misma.

En esta etapa se identifica y define cada una de las actividades de los centros, el proceso se completa en esta etapa con la distribución o reparto de los costos localizados en los centros, entre las distintas actividades que lo han generado.

En esta etapa se han conocido los costos de las actividades, y se ha determinado el portador o generador de costo, el costo unitario se determina dividiendo los costos totales de cada actividad entre el número de generadores de costos. El costo unitario del generador de costos representa la medida de consumo de recursos que cada inductor ha necesitado para llevar a cabo su misión, o en otros términos, el costo que cada inductor genera dentro de una actividad concreta.

En la tercera fase se asignan a los productos los costos de las actividades y además se asignan a esos mismos productos, los costos directos correspondientes. 
Figura 6

Fase 3 Fase Final

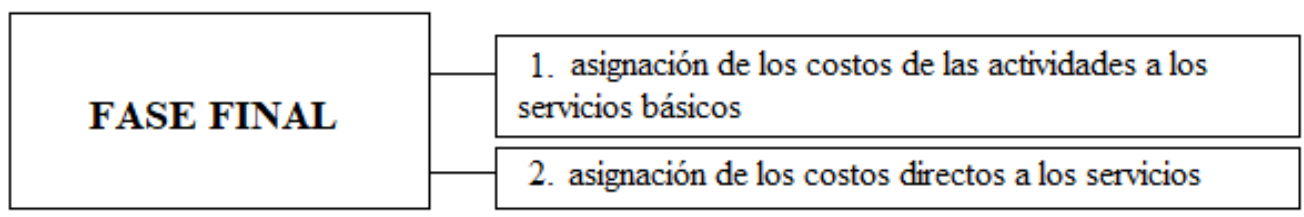

Fuete: Elaboración propia

Esta etapa tiene una gran significación en el modelo $A B C$, pues se sabe que los productos o servicios consumen actividades y las actividades consumen recursos, siendo los generadores o inductores, los que relacionan de manera directa a unos y otros. Llegado este momento en el proceso de asignación, son conocidos ya los costos generados por cada portador de costos; asimismo, y de acuerdo con la correspondencia directa de éstos y los productos, se puede saber de manera inmediata el consumo de cada unidad de servicio básico. El consumo realizado de cada actividad vendrá expresado por el número de prestaciones con las que la actividad ha contribuido a la formación del servicio básico, es decir viene expresado por los inductores o generadores de costos consumidos en los diferentes niveles del proceso de servicio.

El proceso de asignación culminará en esta etapa trasladando los costos directos respecto del servicio en cada uno de ellos. En este modelo los centros de actividades portadores de costos directos, también se van a controlar en actividades, aunque tengan una incidencia directa respecto a los Servicios Básicos. En esta etapa se deben de realizar algunas consideraciones como son:

Para aplicar el sistema de costos $A B C$, al Hotel Panorama lo vamos a distribuir en seis centros de costos, es decir, tres centros de costos productivos (alojamiento, restaurante-bar y salón de eventos), en consecuencia, tres centros de costos de apoyo (administración, lavandería y teléfono).

\section{Información general para determinar los ingresos}

Cuenta con las siguientes habitaciones:

$\begin{array}{lrrr}\text { Sencillas } & 40 \text { habitaciones: disponibilidad } & 950 \\ \text { Dobles } & 32 \text { habitaciones: disponibilidad } & 500 \\ \text { Triples } & 4 \text { habitaciones: disponibilidad } & 100 \\ \text { Total } & 76 \text { habitaciones: disponibilidad } & 1550\end{array}$

Por su parte: El Hotel Panorama cuenta con una disponibilidad de 1550 Habitaciones/Mes, el nivel de ocupación es de $49 \%$. El $60 \%$ corresponde a Habitaciones/Sencillas, el $32 \%$ Habitaciones/Dobles y el $8 \%$ Habitaciones/Triples.

$\begin{array}{lrrr}\text { Tarifa Habitaciones Sencillas } & \$ 100.000 & \\ \text { Tarifa Habitaciones Dobles } & \$ & 200.000 & \\ \text { Tarifa Habitaciones Triples } & \$ & 270.000 & \\ \text { Seguro Hotelero } & \$ & 2.000 \text { por persona }\end{array}$

Recuperación costo de alimentos a empleados: 1000 comidas a $\$ 1500$

Tarifa Alimentación y Bebidas: $\$ 30.000$ por huésped diario

Promedio de ventas diarias del $49 \%$ de ocupación; el $75 \%$ de los huéspedes.

Alquiler por horas de salones de eventos 200.000 por horas (1800 horas/año)

Información de egreso 


$\begin{array}{llrr}\text { Beneficiarios } & \text { Concepto } & \text { Valor } \\ \text { Taller Mabe } & \text { Reparación de estufas } & \$ & 90.000 \\ \text { Libia Tamara } & \text { Capacitación a personal de recepción } & \$ & 140.000 \\ \text { Decoraciones Rosita } & \text { Flores recepción y habitaciones } & \$ & 200.000 \\ \text { Decoraciones Rosita } & \text { Flores para la administración } & \$ & 150.000 \\ \text { Drogas la cascada } & \text { Drogas para el botiquín del área administrativa } & \$ & 30.000 \\ \text { Electro Mabe } & \text { Instalación de máquinas lavadoras } & \$ & 170.000 \\ \text { Fotocopias } & \text { Fotocopias para Administración } & \$ & 25.000\end{array}$

\section{Información para movimientos de inventarios.}

El control de inventarios se hará por medio del sistema permanente en este resumen. determinación los consumos de provisiones y materiales, aplicados a cada departamento. (Inv. inicial + compras - inv final).

Tabla 1

Información de Egreso

\begin{tabular}{lllll}
\hline Clase de Inv. & $\begin{array}{l}\text { Inventario 31 } \\
\text { octubre/2019 }\end{array}$ & $\begin{array}{l}\text { Compras del } \\
\text { periodo: } \\
\text { Contado } 70 \%\end{array}$ & $\begin{array}{l}\text { Inventario 30 } \\
\text { noviembre / } \\
\text { Crédito 30\% }\end{array}$ & consumo \\
\hline $\begin{array}{l}\text { Perecedero } \\
\begin{array}{l}\text { Alimentos } \\
\text { No perecedero }\end{array}\end{array}$ & $\$ 25.600 .000$ & $\$ 14.000 .000$ & $\$ 5.400 .000$ & $\$ 14.200 .000$ \\
bebidas & $\$ 11.000 .000$ & $\$ 22.000 .000$ & $\$ 14.000 .000$ \\
$\begin{array}{l}\text { Mat. Rep. y } \$ 20.000 .000 \\
\text { Accesorio }\end{array}$ & & $\$ 16.000 .000$ & $\$ 4.000 .000$
\end{tabular}

Fuente: elaboración propia

Tabla 2

Consumo de inventario de los diferentes departamentos

\begin{tabular}{|c|c|c|c|c|c|}
\hline Inventarios & Alojamiento & Restaurante & Lavandería & Administración & $\begin{array}{l}\text { Salón de Telefonía } \\
\text { eventos }\end{array}$ \\
\hline Perecederos & $20 \%$ & $60 \%$ & & & $20 \%$ \\
\hline $\begin{array}{l}\text { No } \\
\text { perecederos }\end{array}$ & $11 \%$ & $70 \%$ & & $9 \%$ & $10 \%$ \\
\hline $\begin{array}{l}\text { Mat. Rep. y } \\
\text { Accesorios }\end{array}$ & $40 \%$ & $18 \%$ & $10 \%$ & $20 \%$ & $12 \%$ \\
\hline
\end{tabular}

Fuente: elaboración propia

Información mano de obra

La planta de personal es la siguiente: 
Tabla 3

Planta de personal

\begin{tabular}{|c|c|c|c|c|c|c|c|}
\hline Cargos & Sueldo & Aloja & Rest & Lavan & Admon & Salon E & Telefono \\
\hline Administrador & $\$ 2.500 .000$ & & & & 1 & & \\
\hline Contador & $\$ 1.800 .000$ & & & & 1 & & \\
\hline $\begin{array}{l}\text { Auxiliar } \\
\text { contable }\end{array}$ & $\$ 828.116$ & & & & 1 & & \\
\hline secretaria & $\$ 828.116$ & & & & 1 & & \\
\hline $\begin{array}{l}\text { Jefe } \\
\text { mantenimiento }\end{array}$ & $\$ 828.116$ & & & & 1 & & \\
\hline Botones & $\$ 828.116$ & 2 & & & & & \\
\hline Camarera & $\$ 828.116$ & 2 & & & & & \\
\hline recepcionista & $\$ 828.116$ & 3 & & & & & \\
\hline Chef & $\$ 838.116$ & & 1 & & & & \\
\hline $\begin{array}{l}\text { Ayudante de } \\
\text { cocina }\end{array}$ & $\$ 828.116$ & & 1 & & & & \\
\hline meseros & $\$ 828.116$ & & 2 & & & & \\
\hline $\begin{array}{l}\text { Operario } \\
\text { lavandería }\end{array}$ & $\$ 828.116$ & & & 1 & & & \\
\hline telefonistas & $\$ 828.116$ & & & & & & 3 \\
\hline decorador & $\$ 828.116$ & & & & & 1 & \\
\hline TOTAL & & 7 & 4 & 1 & 5 & 1 & 3 \\
\hline
\end{tabular}

Fuente: elaboración propia

\section{Información de depreciación}

De construcciones y edificaciones: Tiene un área total de $19.000 \mathrm{~m} 2$, distribuida así:

Costo total edificación $\$ 320.000 .000$ de pesos Método depreciación lineal

Tabla 4

Depreciación: Construcciones y edificaciones

\begin{tabular}{lcl}
\hline Departamento & \% del área & vida útil \\
\hline Habitaciones & $75 \%$ & 20 años \\
Restaurante & $12 \%$ & 20 años \\
Lavandería & $3 \%$ & 20 años \\
Administración & $5 \%$ & 20 años \\
Salón de eventos & $5 \%$ & 20 años \\
\hline
\end{tabular}

Fuente: elaboración propia

De equipos de oficina, maquinaria y equipo, flota y equipo de transporte, equipos de computación.

Tabla 4

Depreciación Equipos de oficina, maquinaria y equipo, flota y transporte

\begin{tabular}{llc}
\hline Alojamiento & $v / r$ & da útil años \\
\hline Juego de alcoba & 18.000 .000 & 4 \\
Televisores & 16.000 .000 & 5 \\
Aires acondicionados & 35.000 .000 & 4 \\
\hline
\end{tabular}




\begin{tabular}{|c|c|c|}
\hline Muebles de lobby & 4.000 .000 & 3 \\
\hline Mesas auxiliares & 7.000 .000 & 2 \\
\hline Neveras pequeñas & 15.000 .000 & 5 \\
\hline Total & 95.000 .000 & 23 \\
\hline Restaurante & $v / r$ & vida útil años \\
\hline Equipo de cocción & 15.000 .000 & 2 \\
\hline Terminales de computador & 3.000 .000 & 5 \\
\hline Juego de comedor & 10.000 .000 & 4 \\
\hline Lavaplatos & 1.000 .000 & 10 \\
\hline Equipo de panadería & 4.000 .000 & 5 \\
\hline Cuarto frio & 10.000 .000 & 5 \\
\hline Total & 43.000 .000 & 31 \\
\hline Teléfono & $\mathrm{v} / \mathrm{r}$ & Vida útil años \\
\hline Computador & 10.000 .000 & 3 \\
\hline Escritorios y sillas & 6.000 .000 & 5 \\
\hline Total & 16.000 .000 & 8 \\
\hline Lavandería & $\mathrm{v} / \mathrm{r}$ & vida útil años \\
\hline Lavadora & 10.000 .000 & 5 \\
\hline Secadora & 8.000 .000 & 5 \\
\hline Planchas eléctricas & 6.000 .000 & 2 \\
\hline Carros transportadores & 2.000 .000 & 5 \\
\hline Total & 26.000 .000 & 17 \\
\hline Salón de eventos & $v / r$ & vida útil años \\
\hline Silletería, mesas, manteles & 9.000 .000 & 4 \\
\hline Juego de luces led & 5.000 .000 & 5 \\
\hline Aires acondicionados & 6.000 .000 & 5 \\
\hline Amplificación e Implementos & 5.000 .000 & 5 \\
\hline Total & 25.000 .000 & 19 \\
\hline Administración & $\mathrm{v} / \mathrm{r}$ & vida útil años \\
\hline Escritorios y sillas & 3.000 .000 & 5 \\
\hline Sillas y muebles de oficina & 2.000 .000 & 3 \\
\hline Máquinas registradoras & 1.000 .000 & 6 \\
\hline Cajas fuertes & 1.000 .000 & 20 \\
\hline Archivadores & 2.000 .000 & 15 \\
\hline Vehículo & 30.000 .000 & 5 \\
\hline Equipo de computación & 5.000 .000 & 5 \\
\hline Total & 44.000 .000 & 59 \\
\hline
\end{tabular}


Información de la distribución de los costos a los centros de costos 1

Cuadro 1

Distriución primaria de los costos generales

del hotel distribuidos en centros de costos

\begin{tabular}{|c|c|c|c|c|c|c|}
\hline & \multicolumn{3}{|c|}{ centro de costos productivos } & \multicolumn{3}{|c|}{ centros de costos de apoyo } \\
\hline COSTOS & ALOJAMIENTO & RESTAURANTE & SALON DE EVENTO & LAVANDERIA & TELEFONO & ADMINISTRACIÓN \\
\hline 90.000 & & 90.000 & & & & \\
\hline $\begin{array}{l}\text { capacitacion a } \\
\text { personal de } \\
\text { recepcion } 140.000\end{array}$ & & & & & & 140.000 \\
\hline $\begin{array}{l}\text { flores recepcion y } \\
\text { habitaciones } 200.000\end{array}$ & 200.000 & & & & & \\
\hline $\begin{array}{l}\text { flores para la } \\
\text { admon150.000 }\end{array}$ & & & & & & 150.000 \\
\hline $\begin{array}{l}\text { drogas para el } \\
\text { botiquin del area } \\
\text { admon } 30.000\end{array}$ & & & & & & 30.000 \\
\hline $\begin{array}{l}\text { instalacion de } \\
\text { maquinas lavadoras } \\
170.000\end{array}$ & & & & 170.000 & & \\
\hline $\begin{array}{l}\text { fotocopias para } \\
\text { admon } 25.000\end{array}$ & & & & & & 25.000 \\
\hline
\end{tabular}

Fuente: elaboración propia

Información de la distribución de los costos a los centros de costos 2

Cuadro 2

Distriución primaria de los costos generales

del hotel distribuidos en centros de costos

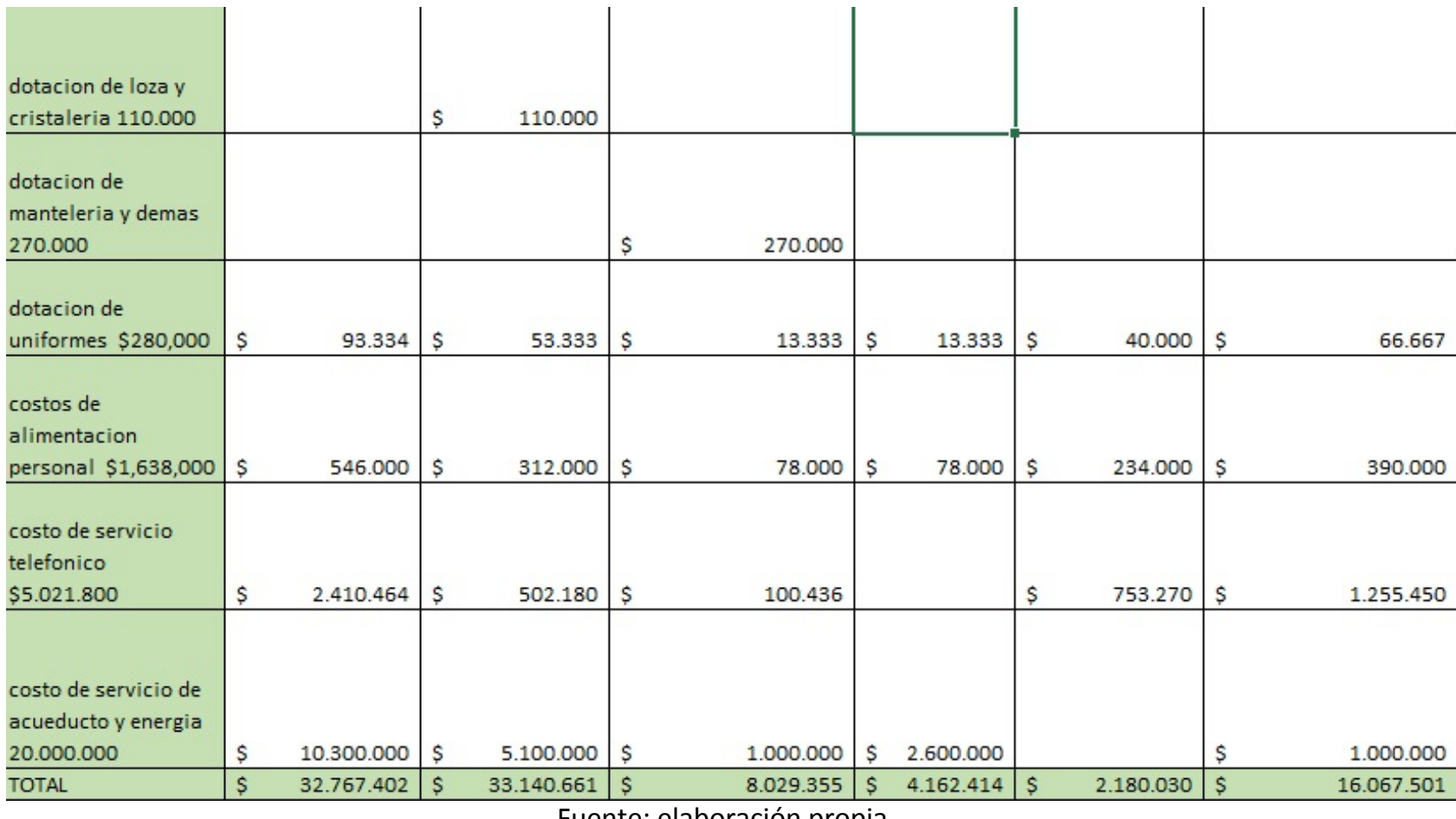

Fuente: elaboración propia 


\section{Resultados}

Las principales ventajas del diseño de un sistema de costos $A B C$ para cualquier empresa es que permite desarrollar una visión horizontal a través del seguimiento del flujo de los procesos en el sentido de poder descomponer los costos de los productos y las actividades que realiza la empresa. De igual manera el sistema de costos $A B C$ permite analizar en forma más realista y profunda, las posibilidades de reducción de costos. Se pasa de un análisis a nivel muy agregado de gastos-productos a un análisis más detallado y que busca el origen de los costos. Veamos parte de los resultados:

\section{Cuadro 3}

Calculo de costos por actividad aplicando el sistema ABC

\begin{tabular}{|c|c|c|c|}
\hline Centros Costos & Alojamiento & Restaurante & Salón de evento \\
\hline costos propios & $\$ 32.767 .402$ & $\$ 33.140 .661$ & $\$ 8.029 .355$ \\
\hline $\begin{array}{l}\text { costos reasignados } \\
\text { cc.lavanderia }\end{array}$ & $\$ 3.329 .932$ & $\$ 416.241$ & $\$ 416.241$ \\
\hline $\begin{array}{l}\text { costos reasignados } \\
\text { cc. tel efono }\end{array}$ & $\$ 1.962 .027$ & $\$ 109.001$ & $\$ 109.001$ \\
\hline $\begin{array}{l}\text { costos reasignados } \\
\text { cc.administracion }\end{array}$ & $\$ 9.640 .500$ & $\$ 3.213 .500$ & $\$ 3.213 .500$ \\
\hline totales costos & $\$ 47.699 .861$ & $\$ 36.879 .404$ & $\$ 11.768 .098$ \\
\hline \multicolumn{4}{|l|}{$\begin{array}{l}\text { Calculo de costos por actividad } \\
\text { aplicando el sistema ABC }\end{array}$} \\
\hline utilización real del servicios/mes & $\begin{array}{r}760 \text { habitaciones } \\
\text { Ocupación /mes }\end{array}$ & 1372 comidas vendidas/mes & 150 horas de alquiler/mes \\
\hline costo unitario por actividad & $\$ 62.763 /$ por noche & $\$ 26.880 /$ comida & $\$ 78.454 /$ por hora \\
\hline tarifas cobradas & $\$ 76.238$ por noche promedio & $\$ 30.000 /$ comida vendida & $\$ 90.000$ cada / hora de alquiler \\
\hline utilidad por actividad & $\$ 127.237$ & $\$ 3.120$ & $\$ 11.546$ \\
\hline $44,70 \%$ & $21,47 \%$ & $10,40 \%$ & $12,83 \%$ \\
\hline
\end{tabular}

Fuente: elaboración propia

Cuadro 4

Modelo tradicional

\begin{tabular}{|l|c|}
\hline \multicolumn{2}{|c|}{ Modelo tradicional de costos } \\
\hline ingresos & $\$ 139.414 .634$ \\
\hline costos & $\$ 96.347 .363$ \\
\hline & \\
utilidad operacional & $\$ 43.067 .271$ \\
$\%$ & $44,70 \%$ \\
\hline
\end{tabular}

Fuente: elaboración propia

En el sentido anterior el Calculo de costos por actividad aplicando el sistema ABC facilita un mayor control para la toma de deciciones ya que nos permite conocer los costos unitarios por cada actividad y al compararlos con las tarifas cobradas nos arroja una utlidad o perdida individualmente, también nos presenta un panorama de como están distribuidos los costos en cada centro de costos que nos sirve para una reducción de costos o reubicacion, por la supresión de las actividades que no agregan valor; especialmente por su vinculación con la técnica de la administración del costo total. 
Otra de las ventajas que permite el establecimiento de un sistema de costos $A B C$ es que mejora la planificación operativa de una empresa pues suministra una abundante información que sirve de guía para varias decisiones estratégicas tales como: fijación de precios; búsqueda de fuentes e introducción de nuevos productos, así como la adopción de nuevos diseños o procesos de fabricación.

En relación al estudio administrativo del Hotel Panorama de la ciudad de Sincelejo, Sucre se pudo observar que debido a la falta de un sistema de costos $A B C$ se hace difícil calcular ciertas tarifas y como consecuencia realizar un análisis comparativo de costos que le permite a la empresa elevar su competitividad y una mejor toma de decisión.

Al aplicar el sistema de costos $A B C$ se observó que el hotel Panorama presenta un un volumen de costos indirectos que pueden entrar a reducir o tranformar en otros costos necesarios como es el costo de marketing, con relación a la depreciación de equipos de oficina, maquinaria, de flota y transporte presenta un elevado deterioro por lo cual se recomienda buscar precios mas razonables de reposición; razón por la cual se pueden hacer mejores licitaciones para conseguir mejores ofertas de precio. Como ejemplo la compra de camas y colchones entre otros enseres de las habitaciones.

Consideramos que con la aplicación del sistema de costos $A B C$ al Hotel Panorama de la ciudad de Sincelejo, Sucre podrá, por un lado, reducir costos innecesarios y por el otro reorientar ciertos costos en el período más adecuado optimizando de mejor manera los períodos vacacionales o de temporada. La puesta en práctica del referido sistema permitirá a futuro calcular de forma más eficiente las tarifas de cada servicio, así como el de contar con un control más efectivo de los inventarios que posee el referido hotel.

De igual manera, y desde el punto de vista gerencial el referido sistema de costos $A B C$ ayudará a los propietarios en la toma de decisión de reducción de costos en momentos en que la afluencia de huéspedes es más reducida ya sea porque no se encuentran en épocas de temporada o por hechos sobrevenidos ajenos a la actividad hotelera de la región. Permanecer a flote en medio de las crisis no es fácil sobre todo si se cuenta con bastante personal que dependa de una empresa hotelera razón por la cual se amerita una correcta y adecuada distribución de la mano de obra y las funciones que desempeñan. Todos éstos beneficios ofrecidos por el sistema de costos $A B C$ mejoraran definitivamente el rendimiento y desenvolvimientos de las actividades y servicios que ofrece el hotel.

\section{Conclusiones}

La implementación del sistema de costeo $A B C$ es muy útil en la etapa de planeación, pues suministra una abundante información que sirve de guía para tomar decisiones estratégicas tales como: fijación de precios; búsqueda de fuentes e introducción de nuevos productos, así como la adopción de nuevos diseños o procesos de fabricación. Así, el poder descomponer los costos de los productos en las actividades que realiza la empresa, permite analizar en forma más realista y profunda, las posibilidades de reducción de costos. Se pasa de un análisis a nivel muy agregado de gastos-productos a un análisis más detallado y que busca el origen de los costos para maximizar las ganancias sin perder calidad en los servicios que se ofertan.

Es por ello que este método constituye un instrumento de ayuda para la gerencia empresarial pues le permite registrar, planear y controlar todas las actividades que se realizan dentro de su empresa logrando reducir los costos indirectos, por la supresión de las actividades que no agregan valor y en especial por su vinculación con la técnica de la administración del costo total. Consideramos que la aplicación del método propuesto definitivamente permitirá, por un lado, satisfacer las necesidades del mercado y las expectativas de futuros clientes y de igual modo, contribuirá con el bienestar de los gerentes y propietarios de las empresas que apliquen el referido sistema de costeo $A B C$. 
El sistemas de costeo $A B C$ y los tradicionales, son complementarios; de igual forma ambos, se soportan en un conjunto de cuentas, registros, informes, formas, procedimientos y normas. Lo importante es que el $A B C$ permite identificar, clasificar, distribuir y asignar los costos de tal forma que se eliminan de su proceso actividades repetitivas o que no generan el valor agregado.

\section{Referencias bibliográficas}

Brimson, J. A. (1991). Activity Accounting: An Activity-Based Costing Approach,. John Wiley \& Sons.

Cruzado, A. (2017). Diseño de un sistema de costos ABC en la FCA de la UNMSM para la mejor estimación de los

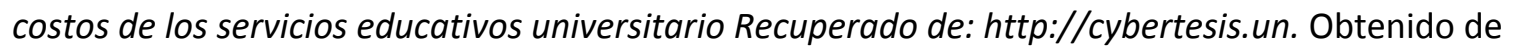
http://cybertesis.un

García , H. M., \& Serrano , P. J. (2006). Costeo basado en Actividades. Revista del Instituto Internacional de Costos, Productividad y Rentabilidad, 18.

Garrido, B. I., Merino, C. L., Morales, M. N., \& Chafla, G. J. (2018). Los sistemas de costos como herramienta fundamental en la administración de empresas. Revista Observatorio de la Economía Latinoamericana. Obtenido de http://www.eumed.net/2/rev/oel/2018/02/costos-administracion-empresas.html

Hernández, R., Fernández, C., \& Baptista, P. (2016). Metodología de la investigación. México D.F: McGraw Hill. Interamericana Editores, S.A. de C.V.

Kaplan, R. S., \& Cooper, R. (1999). Coste y efecto: cómo usar el ABC, AMB y el ABB para mejorar la gestión, los procesos y la rentabilidad (No. 658.15/K17CE).

Laredo, J. B., Martínez, V. F., \& Castañeda, C. E. (2009). XV Congreso internacional de Contaduría, administrativa. Sistema de costos basado en actividades en la fabricación de campanas industriales: aplicación del modelo ABC como herramienta de gestión.

Lawrence, J., \& Chad, J. (2012). Principios de Administracion Financiera. México: Pearson Educacion.México: Pearson Educacion.

Paredes, L. E. (2017). Sistema de costeo basado en actividades (ABC) para la línea de producción de una obra residencia multifamiliar en la constructora "CONSTRUINGENIUM" CIA LTDA de la ciudad de Puyo. PuyoEcuador.

Polimeni , R. S., Fabozzi, F. J., Adelberg, A. H., \& Kole, M. A. (1997). Contabilidad De Costos (3ed). SANTA FE DE BOGOTÁ, D.C. Colombia: (M. E. Suarez R., Ed.) (COST ACCOU). McGRAW-HILL INTERAMERICANA, S.A. Obtenido de https://es.scribd.com/doc/62676837/Contabilidad-de-Costos-3ra-Edicion-Ralph-SPolimeni-P

Ramírez , P. D. (2007). Contabilidad Administrativa, (Decima ed.). México: McGraw Hill.

Esta obra está bajo una Licencia Creative Commons Attribución-NoCommercial 4.0 International

(cc) BY-NC 